\title{
KNOWLEDGE MANAGEMENT AUDIT: A PILOT PROJECT AT A LARGE MULTI-NATIONAL CORPORATION
}

\author{
Jennifer Breese-Vitelli, Robert Morris University, breesevitelli@comcast.net \\ Robert Joseph Skovira, Robert Morris University, skovira@rmu.edu
}

\begin{abstract}
This knowledge management audit explores the best way to harness and house tacit knowledge in a multinational corporation. Connecting people through collaboration helps companies make better/faster decisions, do more with less, speeds the learning process, and improve motivation. Gaining acceptance from employees with regard to best practices is a key component in the implementation of a knowledge management project. Those who will be affected by the change were part of the process of development through the ability to provide input in survey. Input from the impacted population has been bestowed validity to the project. The pilot revealed that the primary reason for hesitation among participants was that others would not do their part in updating the Wiki. Incentive-based compensation is related to the program is suggested to neutralize resistance to participation and foster collaboration.
\end{abstract}

Keywords: Knowledge Management, Information Management, Data Management, Wiki, MIS Behavior

\section{INTRODUCTION}

Knowledge management is defined as the management of organizational knowledge to create business value and generate a competitive advantage (Tiwana, 2002). "Knowledge management is the process by which the organization generates wealth from its knowledge or intellectual capital" (Bukowitz \& Williams, 2000, p. 2). Knowledge management enables the creation, distribution, and exploitation of knowledge to create and retain greater value from core business competencies (Tiwana, 2002). Further, managing knowledge is not about managing the explicit objective, but managing strategy, relationships, and communities of practice, which make up the narrative of the organization (Williams, 2006). Traditionally, knowledge management had been a theory associated with high levels of technology, but the most difficult and often neglected aspect of knowledge management is the cultural shift that is needed to make an organizational change (Hurley \& Green, n.d.). The purpose of this pilot is to develop a blueprint for solving the knowledge management issues that arose when performing a knowledge based SWOT (Strength, Weakness, Opportunity, and Threats) and subsequent knowledge audit to assist in providing a picture of the individual knowledge systems that make up a knowledge platform at a single location of the ABC services operating company comprised of sales professionals. Peter Drucker (1959) stated "plans are only good intentions unless they immediately degenerate into hard work." It is the intention of this paper to develop a plan with a focus on the leadership that is needed to change the culture. The name of the actual company has been changed throughout the paper to maintain its anonymity. The exercises in the study assisted in recognizing: existing knowledge systems, those in need of development, and the cultural issues to be addressed. Also assisting in the audit process to develop the blue print for solving issues that arose was a process detailed by Tiwana (2002) which was used to both identify weaknesses and provide a road map for knowledge management change specifically better knowledge sharing. The Tiwana (2002) ten step process involved four stages similar to those used in this project which are: 


\section{Issues in Information Systems}

Volume 13, Issue 2, pp. 311-319, 2012

Phase 1:

Infrastructure Evaluation

1. Analyze the Existing Infrastructure

2. Align Knowledge Management and Business Strategy

Phase 2:

Align Knowledge Management and Business Strategy

3. Design the Knowledge Management Infrastructure

4. Audit Existing Knowledge Assets and Systems

5. Design the Knowledge Management Team

6. Create the Knowledge Management Blueprint

7. Develop the Knowledge Management System

Phase 3:

Deployment

8. Deploy, Using the Results-driven Incremental Methodology

9. Manage Change, Culture and Reward Structures

Phase 4:

Evaluation

10. Evaluate Performance, Measure ROI, and Incrementally Refine the Knowledge Management System

\section{Introduction/Background Strengths Weaknesses and Threats}

A knowledge management pilot based SWOT, (Strength, Weakness, Opportunity and Threat), was preformed to provide an analysis of the $\mathrm{ABC}$ services corporation which is a part of the umbrella $\mathrm{ABC}$ corporation. The inside sales department a ABC Services division of the ABC Services Operating Company employs 738 employees in Phoenix, Pittsburgh, Memphis, Dallas, New York City, Los Angeles, and San Francisco. ABC Services coordinates sales, marketing and technology support globally for the $\mathrm{ABC}$ brand, the function of the organization is to provide customers with a convenient, single point of access to $\mathrm{ABC}$ shipping, information and supply chain services. $\mathrm{ABC}$ is a publicly traded transportation and logistics company with over 290,000 employees and independent contractors.

The business strategy adopted by $\mathrm{ABC}$ is based on a philosophy of people, service, and profit. This philosophy encompasses a four point strategy: improve service levels, lower unit costs, establish leadership in the international market space, and getting closer to customers. Although the corporate mission is important, it is a rather inclusive statement without many differentiating factors. The corporate strategy is a differentiating statement that stands on its own in describing the mission, direction, and objectives of ABC. The corporate strategy details the desires of company and aims to work seamlessly and independently on three levels. First, operating independently describes the focus placed on the independent networks to meet distinct customer needs. Second, competing collectively is a statement referencing their ability to leverage operating companies under one brand and speak with a collective voice. Lastly, managing collectively is the ability to work together to sustain loyal relationships within the workforce, customers, and investors.

In many ways the strategy of $\mathrm{ABC}$ as an organization is aligned with knowledge management. Tiwana (2002) describes two types of companies that should be pursing knowledge management and ABC fits the description of the first type which is a company that has realized the need to keep up with its competitors and remain a legitimate player in a disruptive highly competitive market place. Major competitors are United Parcel Service (UPS), and DHL a subsidiary of Deutsche Post. ABC was the last to enter into the 


\section{Issues in Information Systems}

Volume 13, Issue 2, pp. 311-319, 2012

competition but the first to reach one billion dollars in annual sales without an acquisition. DHL pulled out of the domestic market competing in the international market space only leaving the market a dualopoly. Because market valuation is largely regarded as an intangible asset ABC leverages its intellectual capital to increase the value investors place on its stock. The ABC brand, similar to companies like McDonalds, uses knowledge management to make policies and appropriate trade-offs in its effort to stay competitive. Trends in the transportation and logistics industry are always changing. The downturn in the shipping of information is due in large part to the current economic recession. The downturn further exacerbated by the also increases in technology and document transfer standards that make delivery unnecessary. Industries ranging from insurance, real estate, to the Federal Government agency the Social Security Administration, have begun to accept electronic transfers and signatures as their regular transfer protocol. Accordingly, the shipment of tangible items has decreased with the downturn of the economy as fewer items are being produced the obvious result being less goods being and shipped. This challenge among the many others in the current economy; the climate at the time of this research is expected to be the most difficult in the company's thirty-eight year history.

ABC, as an organization, fosters collaboration among its employees to gather tacit and explicit knowledge. Tacit knowledge is knowledge that exists in the mind and is difficult to be recorded while explicit knowledge is knowledge that is easily written down. The documentation of the process by which participants are using or developing tools and knowledge is crucial to enable knowledge transfer to others in the organization to: (1) keep the current landscape of underutilization and lack of a value determination from recurring, and (2) retain knowledge lost due to positive and negative attrition. To foster collaboration, the company has regular team meetings to share new technologies and best practices in small groups. Individual teams appoint one Subject Matter Expert (SME) to attend meetings and in turn train their team or disseminate the appropriate information. SME's roll out new technologies and gather input for changes. Ideally, each member of the team is an SME in a particular technology, but it is not unheard of for a team member to be an SME of multiple technologies.

Intellectual capital is protected at $\mathrm{ABC}$ through a variety of security measures. An internal website is dedicated to detailing security protocols and policies. Security concerns are addressed through security tools, education, and a focus on personal responsibility. ABC Information Security has defined ABC Information Security policies and standards to reduce the risk for $\mathrm{ABC}$ and $\mathrm{ABC}$ customers by protecting information assets that are owned and controlled or entrusted to the ABC Corporation. Presentations directed to employees address the challenges of protecting the ABC brand in today's predatory economy, converging public and private information, escalating regulations, growing global infrastructure, owned by each operating company, and increasing visibility as an industry leader. ABC recognizes their strongest partners are their employees and advises them on the creation of user ID and password choices, email, the Internet, instant messaging, data storage, and many other security topics.

$\mathrm{ABC}$ has technologies in place for information management of both internal and external information. New technologies and the agility of the company to be a first adopter of new technologies continues to provide opportunities for $\mathrm{ABC}$; additionally, the ability to build on tracking technologies to include or update existing external technologies like RFID. Ecommerce opportunities such as eBay shippers have been stalled due to contractual issues, although they might be making headway in that arena. ABC always has the option of running subsidiaries together if they are not profitable running separately to provide customers with a wide-breadth of shipping options and a greater customer perception of a united front. Contracts with large companies for example USPS, American Express, Google, and QuickBooks provide opportunities and road maps for continued expansion of these profitable associations and joint program development.

The culture of $\mathrm{ABC}$ is one of pride and value. The company goes to great lengths to instill the goal of the corporate promise to their employees. The promise states that employees will make every experience with $\mathrm{ABC}$ a positive one. Also, employees recognize and embrace the fact that $\mathrm{ABC}$ is viewed as a barometer in 


\section{Issues in Information Systems}

Volume 13, Issue 2, pp. 311-319, 2012

the economy. Articles of fiscal health or difficulties are announced in formal company communication and through informal email communication as soon as the reports are made public. The Principle Accounting Officer of the company has publicly declared that, (50\%) the value of ABC stock is not in assets but rather based on reputation.

\section{SWOT in Terms of Knowledge Management}

In terms of knowledge management there are strengths, weaknesses, opportunities, and threats to $\mathrm{ABC}$. The strengths of ABC knowledge management are their culture and existing security measures. The culture of the organization, the loyalty of their employees and the common understanding of approved communication are its greatest strengths. The security features already in place are strength for $\mathrm{ABC}$; the policies are detailed and the tools developed to protect intranet information are well engineered. Weaknesses include the glut of similar information held in multiple locations. As companies were acquired by $\mathrm{ABC}$ technology their technology systems were not all effectively integrated and as a result the systems still remain disjointed. It is often difficult to find information and when information is conflicting there is no rule of thumb as to which system would trump the other. A cohesive system with better search tools would greatly benefit the company. Opportunities with regard to knowledge management are the ability of $\mathrm{ABC}$ to combine operating companies for better cost efficiency and knowledge transfer. The current divide among the operating companies decreases cost efficiency, and requires duplicate information be stored in multiple systems for retrieval. Threats to the company are the increased use of electronic communication and the freedom with which information is shared. ABC recognizes their greatest partners in security are their employees; however, they also serve as their greatest threat. When rival transportation company DHL experienced financial difficulties their employees blogged about company woes on various public internet sites. Employees provided daily and almost hourly updates of their layoffs and terminal closings which provided competitors with valuable information. Similarly, disgruntled ABC employees could share confidential information and disseminate it quickly in this information age.

\section{SWOT Summary/Conclusion}

The knowledge based SWOT provided a picture of the individual knowledge systems that make up the knowledge platform. ABC is a company that has realized the need to keep up with its competitors and remain a legitimate player in a disruptive market place. The SWOT identified the greatest strength of $\mathrm{ABC}$ as their brand. $\mathrm{ABC}$ was the last to enter the market but, the first to reach one billion dollars in sales without an acquisition. ABC brand identity has been aided by the logo as a symbol of speed and precision that design students revel in studying year after year. The logo has won over forty awards world-wide and continues to gain recognition. The ABC logo can be seen in over 220 countries world-wide and branding campaigns have accompanied their increased role in shipping in China and other international markets. However, marketing alone cannot build a brand; a brand has to bring value. Because market valuation is largely regarded as an intangible asset, $\mathrm{ABC}$ leverages their intellectual capital to increase the value investors place on their stock. The greatest weaknesses were identified as the glut of information housed in multiple locations and disconnected systems and the large amount of tacit information controlled by individual employees. As companies were acquired their systems were not integrated into one overarching system and then applications were added to the separate systems causing the problem to proliferate. Tacit knowledge has increased in the organization as a result of these different systems and a lack of a consistent knowledge database for internal information.

Opportunities for $\mathrm{ABC}$ lie in their constant investment in developing technologies and their willingness to be a first adopter of new technology. Some examples are a type new of RFID (Radio Frequency Identification) that allows the customer to monitor in-flight conditions such as temperature for sensitive packages. Additionally, ABC enlisted new social media forms to market to their customers such as Facebook a number of years ago. Threats to the company are the increased use of electronic communication and the freedom with which information is shared. Industries, such as law firms, insurance 


\section{Issues in Information Systems}

Volume 13, Issue 2, pp. 311-319, 2012

companies, and financial services companies, which had been traditionally the bread and butter of the overnight services are not shipping paper copies as they had in the past and ABC has had to look outside of their traditional industries for new shipping options.

An area identified in the SWOT analysis as having little knowledge sharing of tacit knowledge was the Subject Matter Expert (SME)'s information. On a local district level it was proposed that we address the problem of tacit knowledge and lack of knowledge sharing among the individual teams. The opportunity to address the disjointed systems at the company at large level due to acquisitions was not available. A knowledge audit was employed to address the identified area of knowledge management to be improved by the SWOT; a survey tool was developed as the vehicle for the knowledge audit. The aim of the survey was to gain additional data from existing groups that share resources and further identify opportunities to develop knowledge management. The result of completing a knowledge audit is typically a repository.

\section{Knowledge Audit}

\section{RESULTS}

The goal of the knowledge management audit is to address difficulties in collaboration and communication of Subject Matter Expert information among teams. The scope was limited to specifically address issues with the housing and dissemination of SME knowledge internally throughout the company. A collaborative source of information that Account Executives can consult when a question on a special matter arises is necessary. Some problems cited by AE's with the current system are that they do not know who the SME is for an area, the AE may be out of the office or even no longer with the company. The challenge is to find an efficient way to communicate and update our sales team on SME topics; SME's also need to be accountable for updating the team.

One alternative to finding a solution for this problem based on knowledge management could be updating the team with emails, which is currently done. The problem with this method is people do not save emails, do not know how to file them properly for easy retrieval, or they are disregarded due to the constant glut of emails that come through on a daily basis. The result is that additional time and resources are spent searching for answers to questions that only one person on a team might have. Some of the information might be able to be forwarded from a previous email but often times the information is held in a tacit manner and spoken top of mind from the SME.

The ideal knowledge management system addresses difficulties in collaboration and communication of subject matter expert information among teams. The knowledge management system addressed the current issues with the housing and dissemination of SME knowledge internally throughout the company. Better knowledge management will save time and money on wasted searches and provide a place for easily lost tacit knowledge. It will further protect against loss of information through personnel departures, illness, and discourage the formation of dangerous information silos.

The method selected for auditing ABC's knowledge on processes that need reinforcement is a survey tool (see Appendix A). The survey tool consisted of eight questions three questions had multiple parts and there was a mix of both quantifiable and open-ended questions. The audit identified the areas and processes that benefit from a knowledge management process. The survey tool assisted in developing hindsight, insight, and ultimately foresight. The survey tool was administered to 20 participants in a single day. The participants were all AE's who held one of more SME positions within the sales department in the ABC services operating company of ABC. The survey participants were on separate sales teams within their respective districts; the teams are referred to as the Team A and Team B. The survey was in a paper format and participants were allowed to remain anonymous.

Team A was a little more seasoned with an average of 6.9 years or combined experience compared to 5.6 years of combined ABC experience for Team B. Respondents were asked what they felt was a good 


\section{Issues in Information Systems}

Volume 13, Issue 2, pp. 311-319, 2012

method of exchanging knowledge within their teams; respondents were allowed to make more than one suggestion. The answers varied more among the Team A then they did among Team B. Email and a faceto-face meeting were chosen methods of knowledge transfer among 6 of 10 respondents of the Team A with a Blog, Wiki, SalesPoint, and Go-To-Meeting all getting a vote from 1 in 10.9 of 10 respondents in Team B answered that email was an effective way of knowledge transfer on their team. Face-to-face meetings, and webinars tallied 2 of 10 and a virtual library tallied 1 of 10 . Respondents were asked if they found SME information to be useful. Team A answered 10 of 10 that they found SME information to be useful. Similarly 9 of 10 respondents on Team B found SME information to be useful. The next question was whether the respondents found the SME information difficult to find. The Team A was split down the middle with 5 of 5 determining that they had some difficulties obtaining SME information and 5 of 5 determining that they did not experience difficulties obtaining the SME information. On Team B, 7 of 10 respondents answered that they did not have difficulties obtaining SME information. Problem scenarios listed by the Team A order of their frequency were: not knowing who the SME was, the SME not being updated on the information, the SME was out or not available when a question arose. Team B cited the following answers in order of their frequency were asked about difficulties obtaining SME information: no communication, SME not available, and the SME's were not meeting regularly therefore they were not updated to provide answers. Question 6 was excluded from quantification or coding in that a sample timeframe should have been included in the question to obtain better data. Respondents were asked if an internal Wiki page was developed how likely they would be on a scale of $1-5$ to obtain data and utilize the site. The mode or most frequent response by the Team A was one they would be very likely to utilize and obtain SME information from a Wiki site. The median of the Team A was one with $50 \%$ of the respondents answering one and 50\% answering below one on the scale of likelihood to utilize and obtain information from a Wiki page. The mode of Team B in relation to the question of utilization was also one. The median in the case of Team B was lower with score of 2.5. When asked how likely they would be to participate in updating a Wiki page both teams cited time constraints as the most restrictive factor followed by the hesitation that others would not keep the information updated. Both the Team A and Team B scored a mode or most frequent response of one when answering how likely they would be to update a Wiki page. The average of Team B was slightly lower with a score of 2.4 than the Team A with a score of 2.

$\mathrm{ABC}$ has technologies in place for information management of both internal and external information. New technologies and the agility of the company to be a first adopter of new technologies continue to provide opportunities for $\mathrm{ABC}$; additionally, the ability to build on technologies to include or update existing technologies is an area in which $\mathrm{ABC}$ continues to improve. The knowledge management project has resulted in the suggestion of linking the Team A's internal shared site to leverage existing internal resources to a Wiki site which would allow for the housing and updating for all of the subject matters areas. Housing the information in a knowledge repository like an internal Wiki site ensures the information is easily accessible and searchable; the information will be available even if the AE is out of the office or has chosen to leave the company, decreasing the amount of time it will take to get an answer to a question in a particular subject area. The time to receive an answer would decrease resulting in additional time being spent on sales activities resulting in a positive impact to the bottom line. A knowledge repository is a collection of information that is specific in nature and accessible in the company through Intranet and browser technologies. The knowledge repository will allow knowledge assets to be mobilized rapidly. The constraints of this project are the accountability of the AE's to update their SME areas on a regular basis and the limitations of the current technology. A corresponding reward and recognition program and an addition to the quarterly performance evaluation criteria would need to be established for updating the Wiki site to ensure AE accountability.

\section{Knowledge Management Audit Summary/Conclusion}

Survey results indicated both teams felt similarly with regard to the sharing of SME information $50 \%$ of the respondents cited trouble obtaining SME information and 50\% did not. Although not heavily cited as the preferred method of communication among the teams, the most frequent answer when asked whether the 


\section{Issues in Information Systems}

Volume 13, Issue 2, pp. 311-319, 2012

respondents were likely to use a Wiki page to store and obtain SME information was that they were very likely to use the Wiki page if given the opportunity to store and obtain SME information. A Wiki page is a collaboration website that allows for easy creation and editing of interlinking often data based pages. Wiki means fast in Hawaiian which is indicative of the speed of content and material added to these types of sites. Wiki sites are commonly used for long-term storage and devoted to a specific knowledge area. The most frequent hesitation by the participants with using the Wiki was concern over time constraints followed by the feeling that other SME's would not follow-up with keeping the information updated. The lack of sharing in terms of knowledge contribution is consistent with the findings of Selamat, Abullah and Paul (2006) as the primary reason why this activity falls short in the knowledge management process.

\section{Culture}

The success of a knowledge management culture is largely dependent upon a change in behavior in the organization, (Hurley \& Green, 2004). Changing the culture of the organization will involve both incenting users and dictating their participation in the knowledge management project. The ability of employees to see intrinsic rewards for themselves or the organization is limited, (Hurley \& Green, 2004). Motivation to share information can be enhanced by a contest that will suit the motivations of sales professionals generally; the project can begin with a kick-off contest that offers prizes of either time-off or money. Communications are a key factor in the success of a knowledge management project and assist in the cultural change. To address this issue weekly communications from the knowledge management team will provide both motivation and any additional updates to the project. The requirement of updating the SME Wiki will also be added to SME quarterly performance evaluations in addition to existing performance measures. The process of management reviewing update progress on a quarterly basis will aid in keep the information current.

\section{Measure of Success}

The success for Return On Investment (ROI) will be estimated by determining multiple factors. The factors to determine the marginal cost will be the average salary an SME makes hourly and then estimating the hours the learning curve will take to employ the technology to complete their objective of uploading their SME information. Information organization will be assisted through a knowledge management ontology through a taxonomy as suggested by Holsapple and Joshi (2004) indicating classes for knowledge storage. The minimal cost of developing the technology and continuing are taken into consideration by the base business growth estimated for the SharePoint site, although if the pages created have a heavy graphic focus the allocated server space might need to be expanded. A focus on Hyperlinks should alleviate the need for additional allocation of server space. The return on investment further determines the value of new site by both the organization and the SME's in meeting the goals and objectives (Odom \& Starns, 2003). Factors for ROI will be usage, business value, and usability; usage statistics will be used to determine usage, business process and outcome metrics will be used to measure business value, and usability incorporates factors such as training satisfaction ratings (Odom \& Starns, 2003).

\section{CONCLUSION}

"Knowledge Management - An entity's systematic and deliberate efforts to expand, cultivate, and apply available knowledge in ways that add value to the entity, in the sense of positive results in accomplishing its objectives or fulfilling its purpose" (Holsapple \& Joshi, 2004, p.596). The purpose of this pilot was to develop a knowledge management project that would manage organizational knowledge for creating business value and generating a competitive advantage. Many steps were taken in developing the knowledge management tool including a SWOT analysis to identify a need, an audit to further define the need for a KM project, and ultimately a blueprint for the development of the KM tool. Drucker (2003) stated, "to survive and change, every organization will need to turn itself into a change agent" (p. 3). Becoming a change agent involves a systemic transformation of the organization with regard to how 


\section{Issues in Information Systems}

Volume 13, Issue 2, pp. 311-319, 2012

employees are treated, the continued improvement of products and processes, and the viewing of change as a positive endeavor rather than a threatening one (Drucker, 2003). Most importantly Gardner (2006) stated the most import part of changing people's minds is not to change their perspective but rather to develop understanding to in-turn neutralize resistances. With regard to addressing the Knowledge Management needs of $\mathrm{ABC}$, understanding the main resistance to participation in Wiki based updates is the key factor and should be neutralized through participation incentives.

\section{REFERENCES}

1. Are FedEx and UPS Being Diminished Digitally? -- Seeking Alpha. (n.d.). Retrieved February 16, 2009, from http://seekingalpha.com/article/118086-are-fedex-and-ups-being-diminished-digitally

2. Becerra-Fernandes, I. Gonzalez, A. \& Sabherwal, R. (2004). Knowledge management. Upper Saddle River, NJ: Pearson Prentice Hall.

3. Bukowitz, W. R. \& Williams, R. L. (2000). The knowledge management fieldbook. London: Pearson Education.

4. Davenport, T. H. (with Prusak, L.). (1997). Information ecology: Mastering the information and knowledge environment. New York Oxford: Oxford University Press.

5. Davenport, T. H. and Prusak, L. (2000). Working knowledge: How organizations manage what they know. Boston: Harvard Business School Press.

6. Drucker, P. (2003). Future of Management. Executive Excellence, 20(5), 3.

7. Gardner, H. (2006). Changing minds: The art and science of changing our own and other people's minds. Boston, MA: Harvard Business School Press

8. Holsapple, C. W. \& Joshi, K. D. (2004). A formal knowledge management ontology: Conduct, activities, resources, and influences. Journal of the American Society for Information Science and Technology 55 (7), 593-612.

9. Hurley, Tracey A. \& Green, Carolyn W. (2003). Creating a Knowledge Management Culture: The Role of Task, Structure, Technology and People in Encouraging Knowledge Creation and Transfer. Retrieved October 30, 2004 from cobacourses.creighton.edu/MAM/2005/.../HurleyGreen\%20revision.doc

10. McLeod, Raymond, \& Jordan, Eleanor. (2001). Systems development. NY, NY: John Wiley \& Sons Inc.

11. Odom, Cynthia J., \& Starns, John F. (2003, July 1). Kmworld. Retrieved from http://www.kmworld.com/Articles/Editorial/Feature/How-do-you-measure-success--14844.aspx

12. Selamat, M., Abdullah, R., \& Paul, C. (2006). Knowledge management framework in a technology support envirnment. International Journal of Computer Science and Network Security, 6(8A), 101-109.

13. Tiwana, A. (2002). The Knowledge Management Toolkit: Orchestrating IT, Strategy, and Knowledge Platforms (2nd Edition). Upper Saddle River: Prentice Hall PTR.

14. UPS vs. FedEx -- Analysis of the Two Giants in Package Delivery. (n.d.). Retrieved February 20, 2009, 


\section{Issues in Information Systems}

Volume 13, Issue 2, pp. 311-319, 2012

from http://www.streetauthority.com/cmnts/hps/2007/02-05-package-delivery.asp

15. Williams, R. (2006). Narratives of knowledge and intelligence...beyond the tacit and explicit. Journal of Knowledge Management, 10(4), 81-99.

\section{APPENDIX A}

1. How many years have you been with $\mathrm{ABC}$ ?

2. What do you feel is a good method of exchanging knowledge within your team? why

3. What tool for housing knowledge within our team is most useful?

4. Do you find the SME information to be useful?

\begin{tabular}{|l|l|} 
Yes & No \\
\hline
\end{tabular}

5. Have you ever had difficulty obtaining SME information?

\begin{tabular}{|l|l|}
\hline Yes & No \\
\hline
\end{tabular}

If yes, list two recent problems or scenarios that were responsible for the problem

6. How long does is usually take for you to get an answer from an SME when you have a question?

7. If we developed an internal Wiki page to house SME information how likely on a scale from 1-5 would you be to obtain information and utilize the Wiki site? (Circle your answer - 1 being most likely and 5 being not very likely)

\begin{tabular}{|l|c|c|c|c|}
\hline 1 & 2 & 3 & 4 & 5 \\
\hline
\end{tabular}

8. How likely on a scale of 1-5 would you be to participate in updating the Wiki tool with your own SME Information? Why or why not?

\begin{tabular}{|l|l|l|l|l|}
\hline \hline 1 & 2 & 3 & 4 & 5 \\
\hline
\end{tabular}

